\title{
Improving hospital-based point-of-care ultrasound cleaning practices using targeted interventions: a pre-post study
}

\author{
Daniel Van Kalsbeek ${ }^{1^{*}} \mathbb{D}$, Karl Enroth ${ }^{1}$, Elizabeth Lyden², Mark E. Rupp ${ }^{3}$ and Christopher J. Smith ${ }^{4}$
}

\begin{abstract}
Background: Point-of-care ultrasound (POCUS) devices are becoming more widely used in healthcare and have the potential to act as fomites. The objective of this project was to study the thoroughness of cleaning of POCUS machines before and after a quality improvement initiative. We designed a mixed-methods, pre/post study which took place over the course of one year at a university-affiliated health center. Cleaning rates of four ultrasound machines used by hospital medicine and critical care medicine services were evaluated using fluorescent marking. Interventions targeted physicians' knowledge of best practices and improved access to cleaning supplies. Pre- and post-intervention cleaning rates were compared using a generalized linear model. The impact of the corona virus disease of 2019 (COVID-19) pandemic on baseline cleaning rates was also evaluated. Physicians'attitudes and knowledge of cleaning practices were evaluated via unpaired pre/post surveys.

Results: There was significant improvement in thoroughness of cleaning following intervention (pre 0.62, SE 0.05; post 0.89, SE 0.07$), p<0.0001)$. There was no difference in baseline cleaning rates before $(0.63, \mathrm{SE} 0.09)$ and after $(0.61$, SE 0.1 ) the onset of the COVID-19 pandemic ( $p=0.78$ ). Post-intervention surveying found improved understanding of guideline-based cleaning practice, better performance on knowledge-based questions, and fewer reported barriers to machine cleaning.
\end{abstract}

Conclusion: Thoroughness of cleaning of POCUS machines can be improved with practical interventions that target knowledge and access to cleaning supplies.

Keywords: Point-of-care ultrasound (POCUS), Infection control, Cleaning, Disinfection, Quality improvement

\section{Background}

Over the past three decades, the use of point-of-care ultrasound (POCUS) has been increasingly integrated into routine clinical practice, including critical care and hospital medicine [1-3]. Recently the Alliance for Academic Internal Medicine has advocated for POCUS training across all levels of medical education [3]. Advances in technology have allowed ultrasound machines to become more portable and user-friendly, making it possible to

\footnotetext{
*Correspondence: vankalsbeek@live.com

1 Department of Internal Medicine, 982055 Nebraska Medical Center,

Omaha, NE 68198-2055, USA

Full list of author information is available at the end of the article
}

perform exams on multiple patients in a relatively short time frame [2, 4]. Clinically relevant infectious organisms have been cultured from ultrasound probes and gels, even when they are not visibly soiled, including methicillin-resistant Staphylococcus aureus, Pseudomonas aeruginosa and vancomycin-resistant enterococci [5-9]. Additionally relevant to the present time, it has been discovered that SARS-CoV-2 may remain viable on plastic surfaces for up to $72 \mathrm{~h} \mathrm{[10].} \mathrm{Thus,} \mathrm{POCUS} \mathrm{has} \mathrm{the} \mathrm{poten-}$ tial to act as a fomite for pathogens which are known to prolong hospital stays, escalate healthcare costs, and increase mortality [11].

Cleaning and disinfection of ultrasound equipment has been shown to significantly reduce microbiological 
burden $[12,13]$. Multiple professional groups have published guidelines for cleaning and disinfecting POCUS machines, and, though difficult to prove due to study design, adherence to these guidelines should reduce the risk of transmitting clinically relevant pathogens [14-21]. Unfortunately, surveys suggest suboptimal cleaning practice and a perceived lack of guidance among ultrasound users [22-24]. Hospital-based studies have shown that environmental cleaning performance can be improved using education and feed-back, leading to reduced likelihood of culturing clinically relevant organisms [20, 21, 25-27]. Similar studies pertaining to point-of-care ultrasound are lacking. To address this, we designed a project with the goal of identifying barriers to guideline-based cleaning of POCUS devices and improving cleaning practices using targeted interventions.

\section{Methods}

\section{Design and setting}

A mixed-methods, pre/post-study of POCUS machine cleaning practices was conducted over a 1-year period at a 718-bed academic health center. This project was considered quality improvement by the local institutional review board and therefore did not require approval as human subject research. Two Philips SPARQ (Andover, MA) and 2 GE Healthcare Venue (Chicago, IL) machines were included in the study. SPARQ machines were primarily used by hospital medicine (HM) faculty and internal medicine (IM) residents. One SPARQ machine was stored on an inpatient medical-surgery unit and the other was stored in a HM work room. Venue machines were used primarily by IM residents, pulmonary/critical care medicine (PCCM) fellows, and PCCM faculty, but they were also accessible to surgical and anesthesia critical care providers. One Venue was stored in a neuro-critical care unit and the other was stored in a medical critical care unit. All machines were outfitted with linear, convex, and phased array transducers.

The IM residency program has approximately 95 house officers, including categorical, preliminary, primary care, and medicine-pediatric residents. The residency program has a longitudinal POCUS curriculum, including workshops, lectures, and a dedicated POCUS rotation. There are 13 PCCM fellows, who also have a dedicated POCUS curriculum and rotation. POCUS training and clinical use is variable for HM and PCCM faculty, with some high-frequency users and some non-users.

\section{Intervention}

Interventions were guided by a targeted needs assessment. To better educate physicians, we created a website that outlined best practices for POCUS cleaning and disinfection [28]. The website included a 3-min instructional video, a summary of cleaning steps, and links to bestpractice guidelines from our institution and relevant professional societies. The website was distributed via email to all resident, fellow, and faculty physicians targeted by our survey. The instructional video was also integrated into training sessions for the IM residents and PCCM fellows. Placards were placed on POCUS machines which included a summary of cleaning steps and a QR code to access the website for just-in-time training.

The educational intervention promoted a 2-step cleaning and disinfection process based on American Institute of Ultrasound in Medicine guidelines [14]. First, users were instructed to remove all visible soiling with a disinfecting wipe or soapy washcloth. Then, using a fresh disinfecting wipe, all high-use areas (touch screen, keyboard, handles, probes, and cords) were wiped thoroughly. PDI Sani-Cloth ${ }^{\circledR}$ AF-3 disinfecting wipes were used throughout the study. This product is a quaternary ammonium-containing, low-level disinfectant, which is appropriate for disinfecting medical equipment that contacts intact skin [29].

We improved access to cleaning materials by ensuring that each machine always had a dedicated container of disinfecting wipes. Machines with inadequate space for wipes were equipped with commercially available canister caddies (Fig. 1). Wipes were restocked by researchers twice weekly, as needed, during study periods. Providers were also encouraged to replace wipes when necessary.

\section{Data collection}

A fluorescent marking gel (DAZO, ECOLAB) was used to monitor POCUS machine cleaning practices. The use of these gels is a well-established method for monitoring environmental cleaning practices in infection control research [25-27, 30, 31]. Circular marks roughly $2.54 \mathrm{~cm}$ in diameter were placed on 12 high-touch areas on each machine including probes, cords, and user-interface surfaces (Figs. 1 and 2). The marking sites were determined based on best practice recommendations $[14,15]$ and guidance from our infection control department. The size of gel markings were informed by pilot testing which revealed this pattern allowed the most consistent application of gel. Gel markings were evaluated twice per week during the first two weeks of each month during the study period by members of the research team (DV and KE). The absence of fluorescence under UV light was classified as cleaned. The presence of any visible fluorescence was classified as dirty. This was done to minimize subjectivity when interpreting partially cleaned marks and because residual fluorescence was felt to indicate poor cleaning technique. The presence of gross debris, defined as the presence of soiling that was visible to the naked eye, was also documented. The machines were 


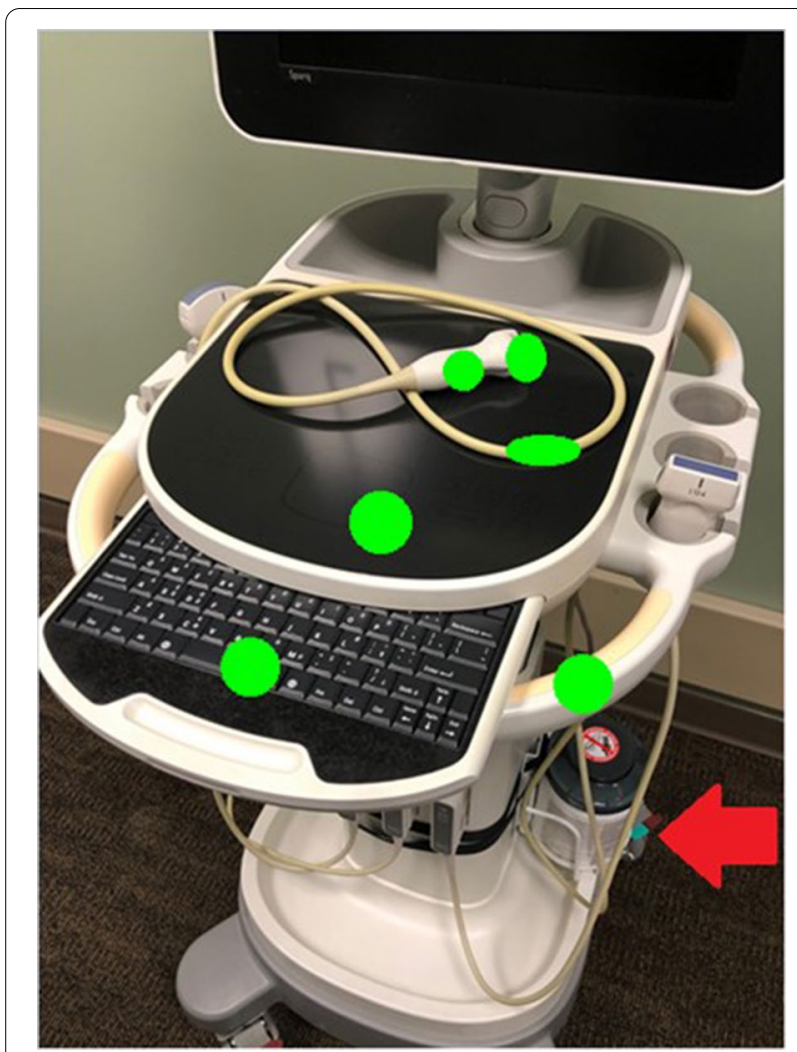

Fig. 1 Locations of fluorescent marks. A Phillips SPARQ model ultrasound machine is pictured to demonstrate marking locations. Green circles represent the areas marked with fluorescent gel during the study (size exaggerated for clarity). For simplicity, only one probe and cord is displayed, but all probes and cords were marked in this fashion during the study. Also pictured is the disinfecting wipe caddie that was added to these models to improve access to cleaning supplies (red arrow)

thoroughly cleaned per institutional guidelines and evaluated with UV light to ensure no remaining fluorescence remained prior to re-marking. Prior to the start of the project, researchers met to ensure machine marking and data collection procedures were consistent between team members. Machine use was tracked via a dedicated signout sheet for each machine.

The pre-intervention data collection occurred from November 2019 to January 2020. Before implementation of the planned intervention, the COVID-19 pandemic spread rapidly across the United States. The heightened awareness around infection control during this time had the potential to confound post-intervention data. To address this possibility, the research team collected an additional 2 months of baseline data from May to June 2020. During this time only three of the four POCUS machines were available for data collection. Interventions were implemented in July 2020. Post-intervention data were collected from August to October 2020.

\section{Survey}

A 16-question survey was created to assess participants' demographics, POCUS experiences, and attitudes and knowledge towards machine cleaning (Additional file 1). The survey was created through an iterative process with input from a survey design expert within the institution. It was pilot tested by three members of a POCUS interest group (one faculty, two residents) to ensure clarity, ease of use, and face validity. The survey was distributed to all IM residents; fellows from PCCM \& Rheumatology; and faculty from HM, PCCM, and Rheumatology with POCUS training. Rheumatology physicians were included as they routinely use POCUS in clinical practice at our institution. A link to the anonymous, online survey was distributed via institutional email in April 2020 (preintervention) and October 2020 (post-intervention).

\section{Analysis}

The primary outcome was the composite machine cleaning rates in the pre-intervention phase compared to the post-intervention phase. Secondary outcomes included comparison of cleaning rates based on primary machine ownership (HM vs PCCM) and marking site groups (transducers vs. cords vs. touch surfaces). Cleaning rates were analyzed using a generalized linear mixed model (GLMM) which accounted for the correlation of cleaning rates for each machine. Model-adjusted mean percentages and standard errors of cleaned components by phase were used to summarize the data. To account for occasions when a machine was not used between sampling periods, data were excluded when there was no documentation of use on the machine's sign-out sheet and there was no evidence of use (i.e., all gel marks remained intact and no gross debris was present). This was done to avoid falsely under-estimating cleaning rates. Unpaired pre- and post-intervention survey results were compared using descriptive statistics. All analyses were done using SAS version 9.4, and a $p$-value of less than 0.05 was considered statistically significant.

\section{Results}

There was no difference in pre-intervention cleaning rates before (0.63, SE 0.09) and after (0.61, SE 0.1) the COVID19 outbreak $(p=0.78)$. These data were, therefore, combined to form an aggregate baseline cleaning rate that was used in the final analysis. Pre- and post-intervention cleaning rates are displayed in Table 1. A total of 768 surfaces were evaluated in the pre-intervention phase (504 pre-COVID, 264 post-COVID) and 504 surfaces in the post-intervention phase. There was a significant improvement in the thoroughness of cleaning following intervention (pre 0.62, SE 0.05; post 0.89 , SE $0.07 ; p<0.0001$ ), 

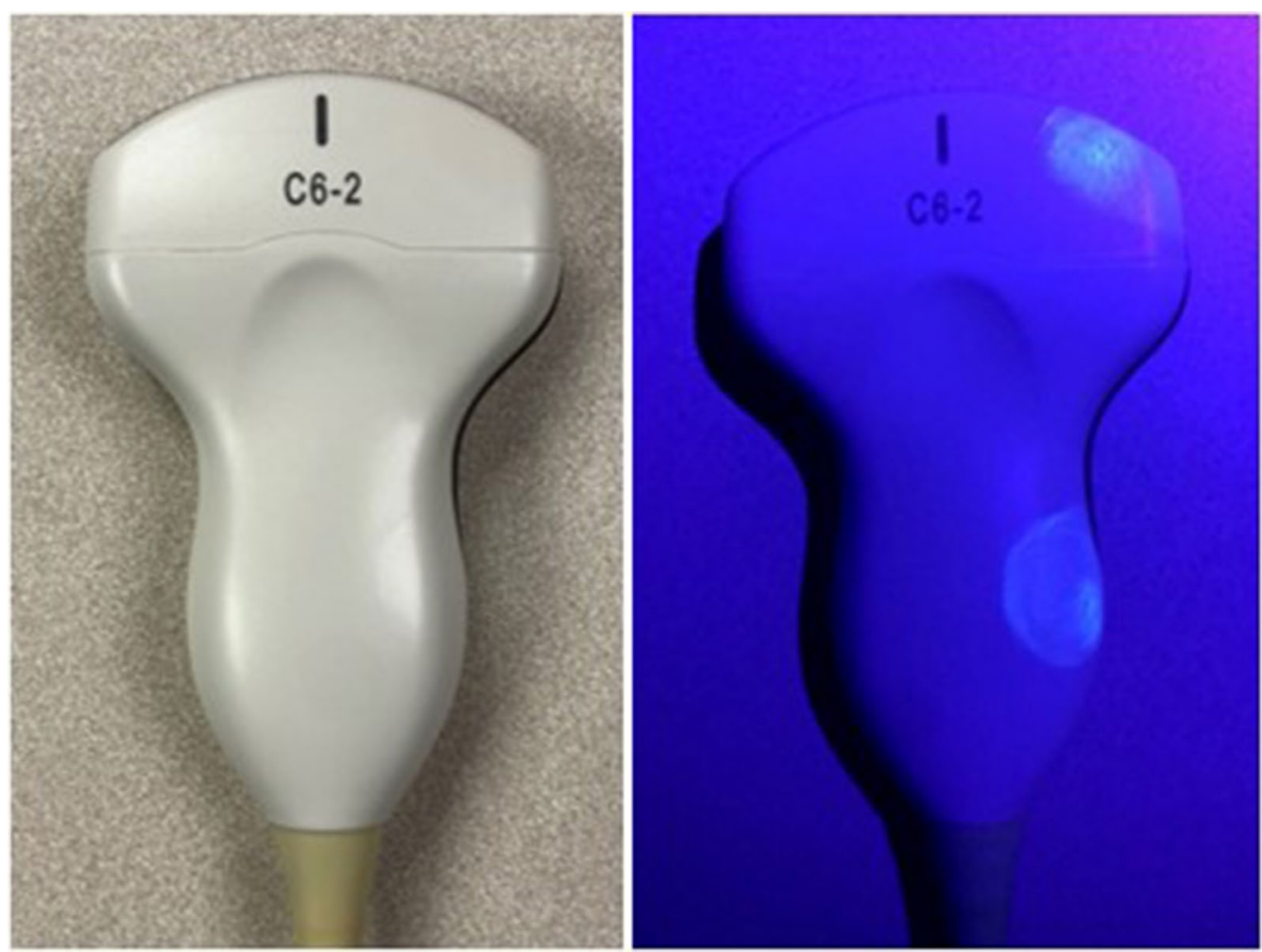

Fig. 2 Fluorescent gel marks. Pictured is a curvilinear probe used to exemplify the size and location of fluorescent marks under ambient room light (left) and UV light with room lights turned off (right)

Table 1 Model-adjusted ultrasound machine cleaning rates before and after quality improvement interventions

\begin{tabular}{lccc}
\hline & Before (SE) & After (SE) & $p$-value \\
\hline All machines combined & $0.62(0.05)$ & $0.89(0.07)$ & $p<0.0001$ \\
Primary machine usage sub-groups & & \\
Hospital medicine machines & $0.50(0.08)$ & $0.91(0.14)$ & $p<0.0001$ \\
Critical care machines & $0.74(0.04)$ & $0.87(0.05)$ & $p=0.075$ \\
Surface category sub-groups & & & \\
Probe lens and handles & $0.74(0.06)$ & $0.96(0.07)$ & $p<0.0001$ \\
Probe cords & $0.40(0.06)$ & $0.78(0.10)$ & $p<0.0001$ \\
High-touch surfaces & $0.63(0.06)$ & $0.88(0.08)$ & $p=0.011$ \\
\hline
\end{tabular}

representing a $44 \%$ relative improvement. In a sub-group analysis, the HM-based machines (pre 0.50, SE 0.08; post 0.91 , SE $0.14 ; p<0.0001$ ) demonstrated greater improvement in the thoroughness of cleaning than the CCMbased machines (pre 0.74, SE 0.04; post 0.87, SE 0.04; $p=0.075$ ). All surface category sub-groups showed significant improvement in cleaning rates $(p \leq 0.01$ for all groups), with probe cords showing the largest improvement (Table 1). During the pre-intervention phase there were 12 instances where gross debris was found on the machines, while there was a single instance in the postintervention phase. All instances of gross debris were found on probe lenses and/or handles. While not specifically analyzed, the gross debris visually appeared to be an accumulation of dried gel, often associated with a designed crevice such as the probe orientation marker. There was a single instance where blood appeared to be intermixed in the gross debris.

Table 2 displays the pre- and post-intervention survey results. The response rates for the pre- and post-intervention surveys were $54 \%(74 / 137)$ and $42 \%$ (58/139), respectively. The majority of respondents were IM residents (66\%). Roughly half of respondents used POCUS weekly or more. Lack of knowledge, limited access to cleaning supplies, and time constraint were identified as the most common barriers to guidelines-based POCUS cleaning and disinfection. Following the intervention, self-reported familiarity with machine cleaning best practices improved from 22 to $74 \%$. The proportion of participants correctly answering knowledge-based questions related to disinfectant use (pre-59\%, post-74\%) and cleaning processes (pre-76\%, post-93\%) also improved. Participants reported fewer barriers related to 
Table 2 Ultrasound machine cleaning survey results before and after quality improvement interventions

\begin{tabular}{|c|c|c|}
\hline Survey topic & Before $(n=74)$ & After $(n=58)$ \\
\hline Female sex, $n(\%)$ & $35(47 \%)$ & $23(40 \%)$ \\
\hline \multicolumn{3}{|l|}{ Level of training, $n(\%)$} \\
\hline Internal medicine resident & $51(69 \%)$ & $36(62 \%)$ \\
\hline Fellow & $6(8 \%)$ & $7(12 \%)$ \\
\hline Faculty & $17(23 \%)$ & $15(26 \%)$ \\
\hline \multicolumn{3}{|l|}{ Faculty/fellow area of practice, $n(\%)$} \\
\hline Hospital medicine/general internal medicine & $9(39 \%)$ & $9(41 \%)$ \\
\hline Pulmonary/critical care & $8(35 \%)$ & $4(18 \%)$ \\
\hline Rheumatology & $6(26 \%)$ & $9(41 \%)$ \\
\hline \multicolumn{3}{|l|}{ Frequency of POCUS use, $n(\%)$} \\
\hline Weekly or more & $34(46 \%)$ & $25(43 \%)$ \\
\hline Less than weekly & $40(54 \%)$ & $33(57 \%)$ \\
\hline $\begin{array}{l}\text { Respondents reporting familiarity with best practice guidelines for POCUS cleaning, } n \\
(\%)\end{array}$ & $16(22 \%)$ & $43(74 \%)$ \\
\hline $\begin{array}{l}\text { Respondents reporting they know where to find institutional guidelines for cleaning, } n \\
(\%)\end{array}$ & $3(4 \%)$ & $33(57 \%)$ \\
\hline \multicolumn{3}{|l|}{ My cleaning practices follow best practice guidelines, $n(\%)$} \\
\hline Agree or strongly agree & $12(16 \%)$ & $34(76 \%)$ \\
\hline Disagree or strongly disagree & $4(5 \%)$ & $0(0 \%)$ \\
\hline I don't know & $58(78 \%)$ & $14(24 \%)$ \\
\hline \multicolumn{3}{|l|}{ Correct response to objective knowledge questions, $n(\%)$} \\
\hline Appropriate use of disinfectants & $44(59 \%)$ & $43(74 \%)$ \\
\hline Recommended steps of cleaning and disinfecting process & $56(76 \%)$ & $54(93 \%)$ \\
\hline \multicolumn{3}{|l|}{ Barriers to cleaning, Median Likert (IQR) ${ }^{a}$} \\
\hline Access to cleaning supplies & $3(2-3)$ & $2(1-3)$ \\
\hline Time constraint & $3(2-3)$ & $2(1-3)$ \\
\hline Lack of knowledge & $3(3-4)$ & $2(1-3)$ \\
\hline
\end{tabular}

a 5 -point Likert scale: $1=$ never, $2=$ rarely, $3=$ sometimes, $4=$ often, $5=$ always

cleaning supply access, time constraints, and knowledge deficits following the intervention.

\section{Discussion}

We were able to significantly improve the thoroughness of cleaning of POCUS machines by using targeted interventions. We identified lack of knowledge, inadequate access to cleaning supplies, and time constraint as primary barriers to guideline-based cleaning and disinfection. These findings support the concern raised by a global survey of ultrasound users which suggested that real-world cleaning practices are unsatisfactory and that users are unfamiliar with guidelines [22, 23]. Given the morbidity, mortality, and cost associated with nosocomial infection, preventative efforts should be pursued whenever feasible [32, 33]. The interventions we described are practical and could be readily implemented at other institutions. A review of guideline-based cleaning and disinfection practice should become a standard component of POCUS training. The effort and expense of such interventions is likely justified if they can prevent even a small number hospital acquired infection [32].

The spread of the COVID-19 pandemic during our preintervention phase had the potential to confound our results, for example, through heightened provider awareness of disinfecting practices or system-level interventions. However, we did not find any difference in cleaning rates following the first spike of COVID-19-related admission in our region of the country [34]. This finding is a powerful example of how difficult it can be to change clinical practices. We believe our project was successful because it identified specific physician-reported barriers allowing for tailored intervention [35].

When sub-grouped by area of use, the thoroughness of cleaning for machines used primarily for critical care approached, but did not meet, significance. These machines are shared with specialties that were not targeted by our interventions, providing a possible explanation for this finding. Of the surface category sub-groups, probe cords had the lowest baseline cleaning rate and the greatest relative improvement. While probe cords 
may not be thought of as a high-contact surface, they are frequently handled during exams or draped across the patient, and attention must be given to this area during cleaning. Though statistical analysis was not performed due to the unpaired nature of our survey, there was an improvement in all response categories following intervention, further supporting the efficacy of these actions.

Although our intervention focused on POCUS users, we would like to highlight an opportunity for manufacturers to improve device design to promote easier cleaning and better infection control. Specifically, there should be efforts to reduce the number of difficult-to-clean crevices, such as we encountered around probe orientation markers. These are in the highest-contact region and we consistently found them to be poorly cleaned. Additional areas of concern include the keyboard and control knobs/ wheels.

There were limitations to our study. This was a singlecenter study, which may limit generalizability. The pre/ post-intervention study design makes it possible that confounding variables influenced our findings. Our design allowed measurement of cleaning but not disinfection, although prior infection control studies demonstrated that improvement in thoroughness of cleaning correlated closely with microbiological assessment [20, 21, 26]. Additionally, thoroughness of cleaning was assessed twice per week, making it possible that machines were used multiple times between assessments. This could have falsely increased our cleaning rates, though should not affect the relative change. Despite these potential limitations, we feel our findings provide meaningful and useful data.

Based on the results of this study, guideline-based cleaning practices and education have been integrated into our POCUS curriculum. This includes ongoing use of the online resources created for the project and improved access to dedicated disinfecting wipes on POCUS machines. In the future, we would like to work with our infection control division to update institutional cleaning and disinfection policies so that they are more easily interpretable with regards to POCUS. An internal sustainability study would add important information, and we would be interested in the findings of a similar project carried out at other sites. Finally, demonstrating a correlation between improvement in point-of-care ultrasound cleaning and decreased patient acquisition of microbes or healthcare-associated infection is desirable.

\section{Conclusion}

We identified barriers to guideline-based cleaning and disinfection of point-of-care ultrasound machines: a lack of knowledge, time constraint, and inadequate access to cleaning supplies. By targeting these domains with practical interventions, we were able to significantly improve the thoroughness of cleaning of four point-ofcare ultrasound machines used by internal medicine and critical care medicine providers.

\section{Supplementary Information}

The online version contains supplementary material available at https://doi. org/10.1186/s13089-021-00244-4.

Additional file 1. Point-of-care ultrasound cleaning survey

\section{Acknowledgements}

The authors would like to thank Dr. Phil Carling for providing DAZO gel, Alisha Dorn for technical infection control expertise, Zachary Benton-Slocum for his work with website creation and design, and Dr. Derek Kruse and Dr. Amy Cannella for being delegates of their respective specialties.

\section{Authors' contributions}

DV: conceptualization, project administration, data collection, data interpretation, media design, media editing, survey design, survey distribution, writing —original draft, writing — review and editing. KE: conceptualization, data collection, data interpretation, media design, media editing, writing — review and editing. EL: statistical analysis, data interpretation, writing - review and editing. MR: conceptualization, data interpretation, writing-review and editing. CS: conceptualization, project administration, project supervision, survey design, survey distribution, data interpretation, writing — review and editing. All authors read and approved the final manuscript.

\section{Funding}

This research did not receive any specific grant from funding agencies in the public, commercial, or not-for-profit sectors.

\section{Availability of data and materials}

The datasets used and/or analyzed during the current study are available from the corresponding author on reasonable request.

\section{Declarations}

Ethics approval and consent to participate Not applicable.

\section{Consent for publication}

Not applicable.

\section{Competing interests}

The authors declare that they have no competing interests.

\section{Author details}

${ }^{1}$ Department of Internal Medicine, 982055 Nebraska Medical Center, Omaha, NE 68198-2055, USA. ${ }^{2}$ Department of Biostatistics, 984375 Nebraska Medical Center, Omaha, NE 68198-4375, USA. ${ }^{3}$ Department of Internal Medicine, Division of Infectious Diseases, 985400 Nebraska Medical Center, Omaha, NE 68198-5400, USA. ${ }^{4}$ Department of Internal Medicine, Division of Hospital Medicine, 986430 Nebraska Medical Center, Omaha, NE 68198-6430, USA.

Received: 10 May 2021 Accepted: 7 October 2021

Published online: 18 October 2021

References

1. Beaulieu Y, Marik PE (2005) Bedside ultrasonography in the ICU: part 1. Chest 128(2):881-895

2. Campbell SJ, Bechara R, Islam S (2018) Point-of-care ultrasound in the intensive care unit. Clin Chest Med 39(1):79-97 
3. Soni NJ, Schnobrich D, Mathews BK et al (2019) Point-of-care ultrasound for hospitalists: a position statement of the society of hospital medicine. J Hosp Med 14:E1-E6

4. Bhagra A, Tierney DM, Sekiguchi H, Soni NJ (2016) Point-of-care ultrasonography for primary care physicians and general internists. Mayo Clin Proc 91(12):1811-1827

5. Frazee BW, Fahimi J, Lambert L, Nagdev A (2011) Emergency department ultrasonographic probe contamination and experimental model of probe disinfection. Ann Emerg Med 58(1):56-63

6. Shokoohi H, Armstrong P, Tansek R (2015) Emergency department ultrasound probe infection control: challenges and solutions. Open Access Emerg Med 7:1-9

7. Marigliano A, D'Errico MM, Pellegrini I, Savini S, Prospero E, Barbadoro P (2010) Ultrasound echocardiographic gel contamination by Burkholderia cepacia in an Italian hospital. J Hosp Infect 76(4):360-361

8. Westerway SC, Basseal JM, Brockway A, Hyett JA, Carter DA (2017) Potential infection control risks associated with ultrasound equipment-a bacterial perspective. Ultrasound Med Biol 43(2):421-426

9. Lee AS, White E, Monahan LG, Jensen SO, Chan R, van Hal SJ (2018) Defining the role of the environment in the emergence and persistence of vanA vancomycin-resistant enterococcus (VRE) in an intensive care unit: a molecular epidemiological study. Infect Control Hosp Epidemiol 39(6):668-675

10. van Doremalen N, Bushmaker T, Morris D (2020) Aerosol and surface stability of SARS-CoV-2 as compared with SARS-CoV-1. N Engl J Med 382(16):1564-1567

11. Cosgrove SE, Qi Y, Kaye KS, Harbarth S, Karchmer AW, Carmeli Y (2005) The impact of methicillin resistance in Staphylococcus aureus bacteremia on patient outcomes: mortality, length of stay, and hospital charges. Infect Control Hosp Epidemiol 26(2):166-174

12. Mullins K, Burnham K, Henricson EK, Cohen S, Fair J, Ray JW (2020) Identification and analysis of bacterial contamination of ultrasound transducers and multiuse ultrasound transmission gel bottle tips before and after the aseptic cleansing technique. J Ultrasound Med 39(10):1957-1963

13. Mullaney PJ, Munthali P, Vlachou P, Jenkins D, Rathod A, Entwisle J (2007) How clean is your probe? Microbiological assessment of ultrasound transducers in routine clinical use, and cost-effective ways to reduce contamination. Clin Radiol 62(7):694-698

14. AIUM official statement: guidelines for cleaning and preparing externaland internal-use ultrasound transducers and equipment between patients as well as safe handling and use of ultrasound coupling gel. American Institute of Ultrasound in Medicine. Updated March 27, 2020 https://www.aium.org/officialStatements/57. Accessed 7 Dec 2020

15. ACEP Policy Statement: Guideline for Ultrasound Transducer Cleaning and Disinfection. Published June 2018. https://www.acep.org/globalasse ts/new-pdfs/policy-statements/guideline-for-ultrasound-transducercleaning-and-disinfection.pdf. Accessed 12 Dec 2020

16. ACEP guideline on COVID-19: ultrasound machine and transducer cleaning. Published March 2020. https://www.acep.org/globalassets/ new-pdfs/policy-statements/guideline-on-covid-19--ultrasound-machi ne-and-transducer-cleaning.pdf. Accessed 12 Dec 2020

17. Basseal J, Westerway S, Juraja M et al (2017) Guidelines for reprocessing ultrasound transducers. Australas J Ultrasound Med 20:30-40

18. Koibuchi H, Kotani K, Taniguchi N (2013) Ultrasound probes as a possible vector of bacterial transmission. Med Ultrason 15(1):41-44

19. Suleyman G, Alangaden G, Bardossy AC (2018) The role of environmental contamination in the transmission of nosocomial pathogens and healthcare-associated infections. Curr Infect Dis Rep 20(6):12
20. Hayden MK, Bonten MJ, Blom DW, Lyle EA, van de Vijver DA, Weinstein RA (2006) Reduction in acquisition of vancomycin-resistant enterococcus after enforcement of routine environmental cleaning measures. Clin Infect Dis 42(11):1552-1560

21. Eckstein BC, Adams DA, Eckstein EC et al (2007) Reduction of Clostridium Difficile and vancomycin-resistant Enterococcus contamination of environmental surfaces after an intervention to improve cleaning methods. BMC Infect Dis 7:61

22. Westerway SC, Basseal JM (2017) The ultrasound unit and infection control-are we on the right track? Ultrasound 25(1):53-57

23. Westerway SC, Basseal JM, Abramowicz JS (2019) Medical ultrasound disinfection and hygiene practices: WFUMB global survey results. Ultrasound Med Biol 45(2):344-352

24. Hoyer R, Adhikari S, Amini R (2016) Ultrasound transducer disinfection in emergency medicine practice. Antimicrob Resist Infect Control 5:12

25. Carling PC, Parry MM, Rupp ME et al (2008) Improving cleaning of the environment surrounding patients in 36 acute care hospitals. Infect Control Hosp Epidemiol 29(11):1035-1041

26. Goodman ER, Platt R, Bass R, Onderdonk AB, Yokoe DS, Huang SS (2008) Impact of an environmental cleaning intervention on the presence of methicillin-resistant Staphylococcus aureus and vancomycin-resistant enterococci on surfaces in intensive care unit rooms. Infect Control Hosp Epidemiol 29(7):593-599

27. Carling PC, Briggs JL, Perkins J, Highlander D (2006) Improved cleaning of patient rooms using a new targeting method. Clin Infect Dis 42(3):385-388

28. Van Kalsbeek D, Enroth K, Benton-Slocum Z, Smith CJ. Cleaning and disinfection of point-of-care ultrasound machines. Published June 2020 https://www.unmc.edu/intmed/residencies-fellowships/cleaning_ultra sound.html. Accessed 24 Jan 2021

29. Rutala WA, Weber DJ (2008) Guidelines for disinfection and sterilization in healthcare facilities. Center for disease control and prevention

30. Rupp ME, Fitzgerald T, Sholtz L, Lyden E, Carling P (2014) Maintain the gain: program to sustain performance improvement in environmental cleaning. Infect Control Hosp Epidemiol 35(7):866-868

31. Carling P (2013) Methods for assessing the adequacy of practice and improving room disinfection. Am J Infect Control 41(5 Suppl):S20-25

32. Hassan M, Tuckman HP, Patrick RH, Kountz DS, Kohn JL (2010) Cost of hospital-acquired infection. Hosp Top 88(3):82-89

33. Westphal GA, Pereira AB, Fachin SM et al (2019) Characteristics and outcomes of patients with community-acquired and hospital-acquired sepsis. Rev Bras Ter Intensiva 31(1):71-78

34. Douglas County Health Department COVID-19 Dashboards (2020) Douglas county health department. https://www.douglascountyhealth.com/ infectious-disease/diseases-and-conditions/2019-novel-coronavirus2019-ncov. Accessed 7 Dec 2020

35. Grol R, Grimshaw J (2003) From best evidence to best practice: effective implementation of change in patients' care. Lancet 362(9391):1225-1230

\section{Publisher's Note}

Springer Nature remains neutral with regard to jurisdictional claims in published maps and institutional affiliations. 